\title{
Switching Portal Technique in Anterior Cruciate Ligament Reconstruction- Use of an Extra Low and Medial Portal
}

\author{
Abhishek Agarwal ${ }^{1 *}$, Ekansh Debuka ${ }^{2}$, Dharmendra Kumar ${ }^{1}$ and Vineet Sharma ${ }^{3}$ \\ ${ }^{1}$ Assistant Professor, Department of Orthopaedics, King Georges Medical University, Lucknow, India \\ ${ }^{2}$ Senior Resident, Department of Orthopaedics, King Georges Medical University, Lucknow, India \\ ${ }^{3}$ Professor and Head, Department of Orthopaedics, King Georges Medical University, Lucknow, India \\ *Corresponding Author: Abhishek Agarwal, Assistant Professor, Department of Orthopaedics, King Georges Medical University, \\ Lucknow, India.
}

Received: June 06, 2019; Published: June 17, 2019

DOI: $10.31080 /$ ASOR.2019.02.0066

\begin{abstract}
Background: Long term success of ACL reconstruction depends on accurate surgical technique of anatomic tunnel placements and proper rehabilitation protocol followed, and for accurate tunnel positioning various arthroscopic landmark should be visible clear with the use of various portals.

Materials and Methods: All 112 surgeries were performed by a single surgeon in King Georges Medical University, Lucknow Orthopedic department Operation theatre during July 2016- December 2018. An extra low and medial portal is created and then portal were switched between anteromedial and low medial ones.

Results and Conclusion: With the use of this portal the average surgical time was observed to be 43 minutes and no malpositioning of tunnel was encountered. This switching portal technique provides an excellent view of the femoral tunnel plot and gives consistent results with minimum complications
\end{abstract}

Keywords: Switching Portal; Residents Ridge; Arthroscope; Working Portal

\section{Introduction}

With the increasing rate of arthroscopic ACL reconstruction being performed everywhere, the incidence of failure and decreased longevity of the reconstructed graft is being encountered very frequently. Although there can be variety of reasons behind this failure- biological failure, new trauma, faulty surgical technique, the most important reason is due to malposition of tunnel placement more frequently that of femoral tunnel $[1,2]$. According to a data from Multicentre ACL revision society (MARS) $80 \%$ of all ACL failure is because of faulty Femoral tunnel [3]. Anatomic ACL reconstruction is now the method of choice as compared to transtibial technique, the later involves tibial tunnel dependant Femoral tunnel placement [4-6]. Every fraction of milimeters is important as far as accurate positioning tunnel placement is concerned for both entry point as well as the correct trajectory. And to get this we need to see whole plot accurately neat and clear as even minor variation in these principles can lead to bad to worse outcomes.
By the use of quadrant method the ACL femoral foot print is located $28-32 \%$ of the long axis of the quadrant and $35 \%$ of the short axis. There should not be any part of tunnel anterior to the resident's ridge $[7,8]$.

The center of the tibial foot print should be at the level of posterior edge of anterior horn of lateral meniscus antero-posteriorly and in between interspinous area (40-60 ratio) mediolaterally $[9,10]$. Surgeons are using standard two portal technique for single bundle ACL reconstruction since time. The purpose of the study is to emphasize the role of an extra low and too medial portal to reconstruct ACL reliably with accurate femur and tibial tunnel positioning

\section{Materials and Method}

The study was conducted over a period of two and a half years during July 2016 to December 2018 in Department of Orthopaedics, King Georges Medical University, Lucknow. All surgeries were performed by single surgeon. 
Surgical technique

Patient positioned supine

$$
\int \begin{aligned}
& \text { (Bolster over OT Table) } \\
& \text { (60 degree flexion at hip and } 90 \text { degree at knee) }
\end{aligned}
$$

Under GA/RA Tourniquet is tightened

] (Surface Marking Done)

High anterolateral visual portal just at the level lower pole of patella lateral to patellar tendon by vertical stab incision and dilating with straight artery

Entry with blunt trochar and cannula to minimize the damage to the articular cartillage

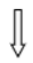

Diagnostic round of the whole knee and confirmation of ACL tear

$\sqrt{ }$

The high anteromedial working portal (which would be switched to visual portal when

doing femoral tunnel) (Figure 1)

II

$3^{\text {rd }}$ a new low and more medial ( $2 \mathrm{~cm}$ to patellar tendon) working portal is made under

arthroscopic vision with spinal needle which would reach the proper femoral foot print

seen through high medial portal

Femoral tunnel is first drilled with guide wire in free hand technique and then drilled

to required diameter according to the diameter of the graft size (keep knee in maximum

flexion to prevent tunnel going too posterior)(care should be taken to ensure at least

$2.5 \mathrm{~mm}$ bony ridge between the tunnel wall and the proximal and posterior wall) it is

important to drill approximately $10 \mathrm{~mm}$ farther to the desired graft length in the

femoral tunnel to allow easy flipping of the endobutton and at least $8 \mathrm{~mm}$ of the lateral

cortex to be intact to prevent tunnel blowout. (Figure 3and 4) (table 3)

$\sqrt{3}$

Tunnel is secured with fiber wire with Beathpin

I

Switching back to anterolateral portal tibial tunnel is drilled usual recommended

technique in outside in fashion(figure-5)

The graft is then passed and endobutton is flipped towards the femoral side and is

secured and tightened with a bioscrew towards the tibial side

\section{Figure}

\section{Result and Discussion}

Total 112 ACL reconstruction in 112 patients with at least 3months post injury instability (Table 1).
Male/female - 90/22

Right/left -- 60/52

Average duration from the time of injury to the reconstruction - 168 days

Single/multiligament -- 103/09

Average time of surgery -- 43 minutes (including the graft harvest and preparation)

Average femoral tunnel length -- $38 \mathrm{~mm}$

Average tibial tunnel length -- $47 \mathrm{~mm}$

Average femoral tunnel diameter $-8.4 \mathrm{~mm}$

Average tibial tunnel diameter -- $9.1 \mathrm{~mm}$

Malpositioning of femoral tunnel-none

Malpositioning of tibial tunnel -none

Femoral tunnel blowout -02

Tibial tunnel blow out -- none

Table 1: Showing the mean observed findings.

Anatomic and accurate positioning of tunnel placement is key to success in ACL reconstruction. Although ACL anatomy has been well described by radiographs and cadaveric measurements but arthroscopic landmarks are of importance in reconstruction. Whole plot should be visible neat and clear to pin Arjuna's fish eye (A reference of accuracy in Indian mythology) and to attain this the use of high anteromedial portal for visualization and extra low medial portal to be used as working portal has been proved to be a very good technique for tunnel placement without necessitating the inadvertent use of notchplasty (figure 2-3). In cases where remnants of ACL hinder the view of footprints, this technique is proven to be superior than two portal technique. In cases where augmentation of ACL is done while preserving the remnant ACL, the use of this extra portal has no match. Snow., et al. has mentioned many advantages of anatomical tunnel placement over the ancient transtibial technique which was further reemphasized by lubovitz [11]. Bedi., et al. has emphasized the importance of accurate tunnel placement on the basis of footprints and landmarks [12]. Paulo., et al. described the three portal technique for double bundle ACL reconstruction. All these statements are in accordance with the technique presented in this study.

The principle for anatomical ACL reconstruction are to functionally reestablish the ACL to its native dimensions, collagen orientation and insertion sites. 


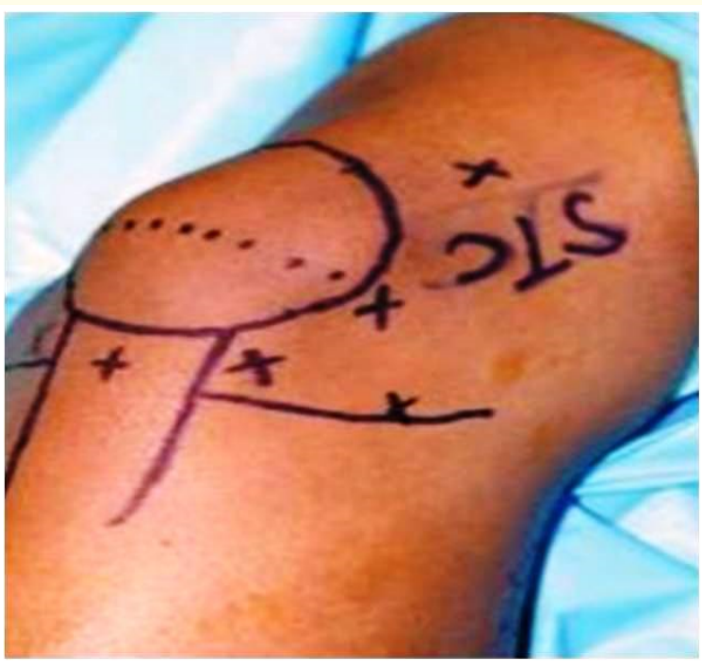

Figure 1: Portal locations marked on skin as for ACL reconstruction.

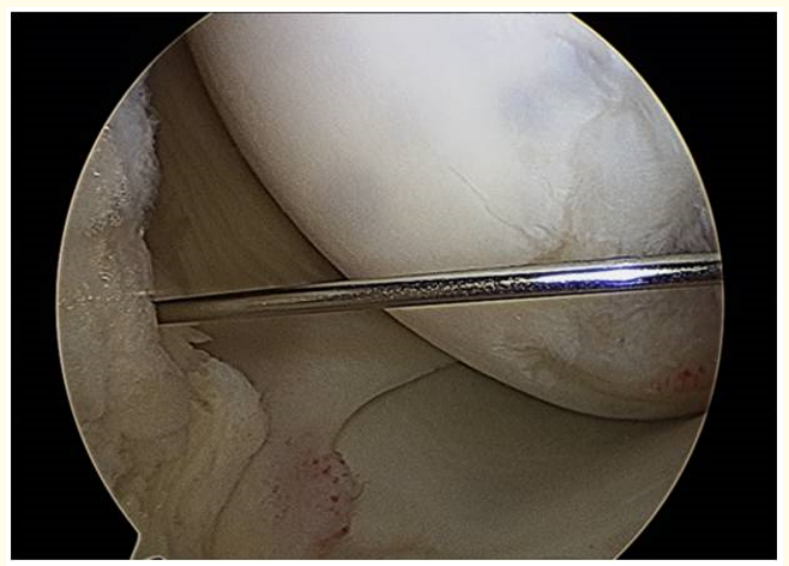

Figure 2: Arthroscope in anterolateral portal.

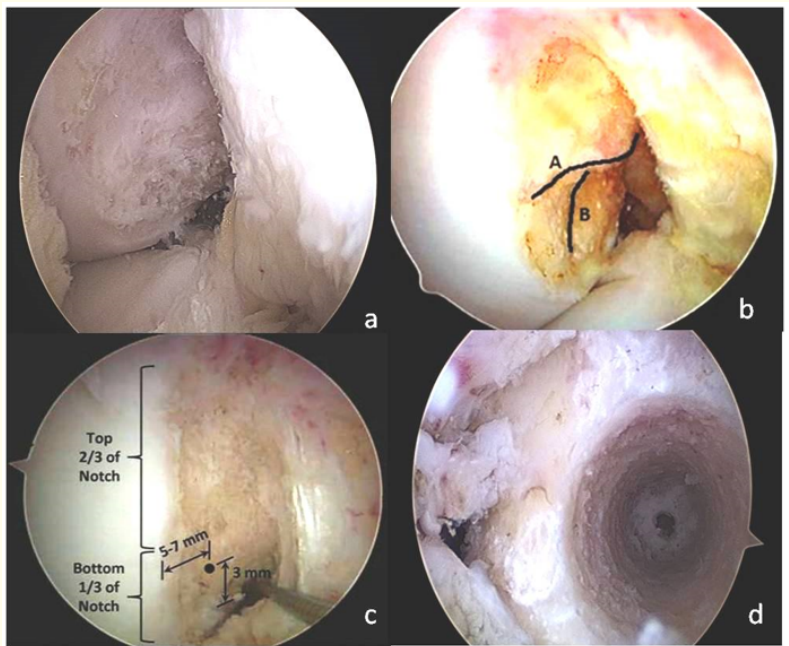

Figure 3: Arthroscope in high anteromedial portal; a. showing medial surface of lateral femoral condyle, b.c.; measurements over lateral femoral condyle, d. final femoral tunnel.

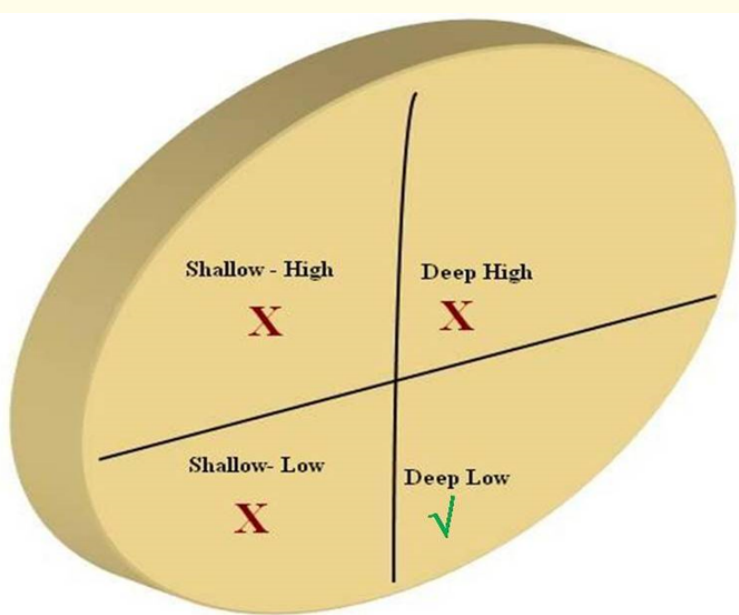

Figure 4: Showing anatomical verses arthroscopic representation (We want femoral foot print to be low and deep).

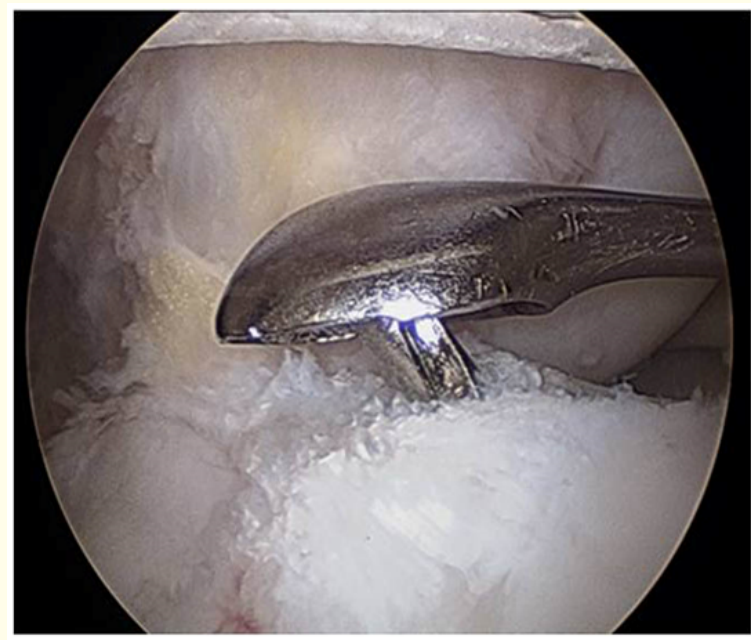

Figure 5: Arthroscope in anterolateral portal showing preparation for tibial tunnel.

\begin{tabular}{|l|l|l|}
\hline & Visual portal & Working portal \\
\hline Diagnostic round & $\begin{array}{l}\text { High antero lateral } \\
\text { portal }\end{array}$ & \\
\hline $\begin{array}{l}\text { Femoral tunnel } \\
\text { placement }\end{array}$ & $\begin{array}{l}\text { High anteromedial } \\
\text { portal }\end{array}$ & $\begin{array}{l}\text { Accessory low medial } \\
\text { portal }\end{array}$ \\
\hline $\begin{array}{l}\text { Tibial tunnel } \\
\text { placement }\end{array}$ & $\begin{array}{l}\text { High antero lateral } \\
\text { portal }\end{array}$ & $\begin{array}{l}\text { High anteromedial } \\
\text { portal }\end{array}$ \\
\hline
\end{tabular}

Table 2: Portal usage according to surgical step in Anatomical ACL reconstruction.

\begin{tabular}{|l|l|}
\hline Anatomical & Arthroscopic \\
\hline posterior & low \\
\hline anterior & high \\
\hline superior & deep \\
\hline inferior & shallow \\
\hline
\end{tabular}

Table 3: Anatomical verses arthroscopic representation. 


\section{Conclusion}

Making of one extra medial portal ( $3^{\text {rd }}$ portal) and switching it as working portal and high medial portal as a visual portal for femoral tunnel placement is a very easy, reproducible and technique which provides a meticulous view of remnants and bony arthroscopic landmarks facilitating anatomical graft placement as compared to two portal technique which limits the proper visualization of femoral insertion site (table 2). In knee arthroscopy any numbers of portals can be made according to your comfort until you make the joint a sieve. The author suggests the use of this accessory anteromedial portal technique for ACL reconstruction.

\section{Bibliography}

1. Ahn J H., et al. "Three-dimensional reconstruction computed tomography evaluation of tunnel location during single-bundle anterior cruciate ligament reconstruction: A comparison of transtibial and 2-incision tibial tunnel-independent techniques". Clinics in Orthopedic Surgery 5.1 (2013): 26-35.

2. Morgan J A., et al. "Femoral tunnel malposition in ACL revision reconstruction”. Journal of Knee Surgery 25.5 (2012): 361-368.

3. MARS Group., et al. "Descriptive epidemiology of the Multicenter ACL Revision Study (MARS) cohort". American Journal of Sports Medicine 38.10 (2010): 1979-1986.

4. Kopf S., et al. "The ability of 3 different approaches to restore the anatomic anteromedial bundle femoral insertion site during anatomic anterior cruciate ligament reconstruction". Arthroscopy 27.2 (2011): 200-206.

5. Tashiro Y., et al. "Comparison of transtibial and transportal techniques in drilling femoral tunnels during anterior cruciate ligament reconstruction using 3D-CAD models". Open Access Journal of Sports Medicine 5 (2014): 65-72.

6. Tompkins M., et al. "Anatomic femoral tunnel drilling in anterior cruciate ligament reconstruction: Use of an accessory medial portal versus traditional transtibial drilling". American Journal of Sports Medicine 40.6 (2012): 1313-1321.

7. Ferretti M., et al. "Osseous landmarks of the femoral attachment of the anterior cruciate ligament: An anatomic study". Arthroscopy 23.11 (2007): 1218-1225.

8. Xu H., et al. "A systematic review of anterior cruciate ligament femoral footprint location evaluated by quadrant method for single-bundle and double-bundle anatomic reconstruction". Arthroscopy 32.8 (2016): 1724-1734.
9. Farrow L D., et al. "Morphology of the femoral intercondylar notch". Journal of Bone and Joint Surgery American 89.10 (2007): 2150-2155.

10. Piefer J W., et al. "Anterior cruciate ligament femoral footprint anatomy: Systematic review of the 21st century literature". Arthroscopy 28.6 (2012): 872-881.

11. Lubowitz JH. "Anteromedial portal technique for the anterior cruciate ligament femoral socket: pitfalls and solutions". Arthroscopy 25.1 (2009): 95-101.

12. Bedi A and Altchek DW. "The Footprint anterior cruciate ligament technique: an anatomic approach to anterior cruciate ligament reconstruction". Arthroscopy 25.10 (2009): 1128-1138.

\section{Volume 2 Issue 7 July 2019}

(C) All rights are reserved by Abhishek Agarwal., et al. 